Courrier du Centre international Blaise Pascal

19 | 1997

Varia

\title{
Bibliographie de Pascal savant
}

\section{Dominique Descotes}

\section{OpenEdition}

Journals

Édition électronique

URL : http://journals.openedition.org/ccibp/561

DOI : 10.4000/ccibp.561

ISSN : 2493-7460

\section{Éditeur}

Centre international Blaise Pascal

\section{Édition imprimée}

Date de publication : 5 avril 1997

Pagination : 17-23

ISSN : 0249-6674

\section{Référence électronique}

Dominique Descotes, «Bibliographie de Pascal savant», Courrier du Centre international Blaise Pascal [En ligne], 19 | 1997, mis en ligne le 06 janvier 2016, consulté le 15 septembre 2020. URL : http:// journals.openedition.org/ccibp/561

Ce document a été généré automatiquement le 15 septembre 2020.

Centre international Blaise Pascal 


\title{
Bibliographie de Pascal savant
}

\author{
Dominique Descotes
}

1 Il existe plusieurs outils bibliographiques sur Pascal savant :

- MAIRE Albert, L'œuvre scientifique de Blaise Pascal. Bibliographie critique et analyse de tous les travaux qui s'y rapportent, Hermann, Paris, 1912, 184 p.

- GOYET Thérèse et HELLER Lane M., Bibliographie Blaise Pascal (1960-1969), Adosa, ClermontFerrand, 1989, $178 \mathrm{p}$.

2 L. M. Heller travaille actuellement à la poursuite de cette bibliographie exhaustive à partir de 1970.

3 Sur les textes particuliers, on trouvera de nombreux titres dans les notices de :

- MESNARD Jean, édition des Euvres complètes de Pascal, vol. 1-4, Desclée de Brouwer, Paris, 1964-1994.

On peut enfin trouver certaines références dans :

- HARA Kokiti, L'œuvre mathématique de Pascal, Memoirs of the Faculty of Letters, Osaka University, vol. XXI, mars 1981, Osaka, p. 231-238.

5 Ne sont mentionnés ici que les ouvrages qui n'ont pas été recensés dans ces recueils, c'est-à-dire soit les plus récents, soit exceptionnellement ceux qui leur ont échappé, et qu'il est utile de signaler.

6 Un même titre a été répété lorsqu'il consacre à un ouvrage ou à un thème une étude particulièrement importante. Nous avons inclus un petit nombre d'ouvrages dont le rapprochement avec des textes pascaliens nous semble s'imposer, comme le livre de Bolzano sur Les paradoxes de l'infini, quoiqu'il n'y soit pas fait expressément mention de Pascal. Cette bibliographie n'est certainement pas complète; tout complément qu'apporteraient nos lecteurs sera accueilli avec reconnaissance et signalé dans une prochaine livraison. 


\section{I/ Pascal mathématicien}

\section{Bibliographie générale}

ALLARD Robert « Pascal et la trente-deuxième proposition d'Euclide », Pascal, Port-Royal, Orient, Occident, Klincksieck, Paris, 1991, p. 191-198.

ALLARD Robert, La jeunesse de Pascal. De la légende à l'histoire, Jean Lagneau, Paris, 1994, 148 p.

BEAUFRET Jean, «Pascal savant », Revue des sciences philosophiques, XL, 1950 ; repris in Dialogue avec Heidegger, II, Minuit, Paris, 1973, p. 54-65.

Blaise Pascal mathématicien, physicien, ingénieur, Ministère de la Culture, Clermont-Ferrand, 1993, $76 \mathrm{p}$.

BOLD Stephen, Pascal geometer. Discovery and invention in seventeenth-century France, Droz, Genève, 1996, 216 p.

BOURBAKI Nicolas, Éléments d'Histoire des Mathématiques, Masson, Paris, 1984, 376 p.

BOY Michel, Pascal, Opuscules philosophiques, Hachette, Paris, 1980, 128 p.

CLERO Jean-Pierre, «Pascal et les mathématiques », in Barbin Évelyne et Caveing Maurice, Les

philosophes et les mathématiques, Ellipses, Paris, 1996, p. 66-88.

COLERUS Egmont, De Pythagore à Hilbert. Les époques de la mathématique et leurs maîtres,

Flammarion, Paris, sd, $312 \mathrm{p}$.

COLLECTIF, Petite Encyclopédie des Mathématiques, Didier, Paris, 1980, 828 p.

COLLETTE Jean-Paul, Histoire des mathématiques, 2, Vuibert-ERPI, Montréal, 1979, 360 p.

COMBE René, « L'œuvre scientifique de Pascal », Nucleus, 1, janv.-fév. 1963, p. 8-23.

COSTABEL Pierre et MARTINET Monique, Quelques savants et amateurs de science au XVII ${ }^{e}$ siècle. Sept notices biobibliographiques caractéristiques, Cahiers d'Histoire et de Philosophie des Sciences, 14, 1986, (Notices sur Roberval, Mersenne, Huygens, Morin, etc.).

DESCOTES Dominique, L'argumentation chez Pascal, P.U.F., Paris, 1993, 464 p.

DHOMBRES J. et SAKAROVITCH J. (dir.), Desargues en son Temps, Blanchard, Paris, 1994, 484 p.

FERREYROLLES Gérard, Les reines du monde. L'imagination et la coutume chez Pascal, Champion, Paris, $1995,316 \mathrm{p}$.

GARDIES Jean-Louis, « Arnauld et la reconstruction de la géométrie euclidienne », in Pariente Jean-Claude (dir.), Arnauld. Philosophie du langage et de la connaissance, Vrin, Paris, 1995, p. 13-32. GRANGER Gilles-Gaston, «L'usage philosophique des mathématiques au XVII ${ }^{\mathrm{e}}$ siècle », Rashed Roshdi (dir.), Mathématiques et philosophie, p. 287-301.

HARRINGTON Thomas More, Pascal philosophe. Une étude unitaire de la Pensée de Pascal, SEDES-CDU, Paris, $1982,190 \mathrm{p}$. 
HEINEKAMP Albert et METTLER Dieter (dir.), Leibniz à Paris (1672-1676), I, Les Sciences, Studia Leibnitiana Supplementa, vol. XVII, Franz Steiner Verlag GMBH, Wiesbaden, 1978, 242 p.

HOGBEN Launcelot, Les Mathématiques pour tous, Payot, Paris, 1962, 688 p.

Inventeurs et Scientifiques. Dictionnaire de biographies, Paris, Larousse, 1994.

Huygens et la France, Vrin, Paris, 1982, 268 p.

ITARD Jean, Essais d'histoire des mathématiques, Blanchard, Paris, 1984, 38 p.

KLINE Morris, Mathematical thought from ancient to modern times, Oxford Univ. Press, 1972, 3 vol.

KLINE Morris, Mathematics. The loss of certainty, Oxford Univ. Press, 1980, 366 p.

LE LIONNAIS (dir.), Les grands courants de la pensée mathématique, Blanchard, Paris, 1962, 560 p.

MESNARD Jean, « Leibniz et les papiers de Pascal », in Heinekamp Albert et Mettler Dieter (dir.), Leibniz à Paris (1672-1676), I, Les Sciences, p. 45-58.

MESNARD Jean, « Les premières relations parisiennes de Christiaan Huygens », Huygens et la France, Vrin, Paris, 1982, 268 p., p. 33-40.

MESNARD Jean, « La science et son ordre. Pascal », Communio, VIII, n 4, juill.-août 1984, p. 77-84. MESNARD Jean, «Sur le chemin de l'Académie des sciences : le cercle du mathématicien Claude Mylon (1654-1660) », Revue d'Histoire des sciences, XLIV, 2, 1991, p. 241-251.

MEURILLON Christian, « La lettre au cœur de l'écriture pascalienne », Revue des Sciences humaines, 195, 1984-3, p. 5-18.

MILHAUD Gérard, « Pascal savant », Europe, 597-598, janv.-fév. 1979, p. 38-53.

RASHED Roshdi (dir.), Mathématiques et philosophie, CNRS, Paris, 1991, 316 p.

ROBERVAL Gilles Personne de, Éléments de géométrie de G. P. de Roberval, éd. V. Jullien, Vrin, Paris, 1996, $544 \mathrm{p}$.

SAUSER Pierre, «L'œuvre mathématique de Pascal et sa place dans les mathématiques du XVII ${ }^{\mathrm{e}}$ siècle ", Mémoires de l'Académie des sciences, et belles-lettres de Dijon, t. 132, 1991-1992, p. 387-394.

SMITH David Eugène, A source Book in mathematics, Dover, New York, 1959, 702 p. (plusieurs textes de Pascal traduits et sommairement commentés).

\section{Introduction à la géométrie}

LEIBNIZ G. W., La caractéristique géométrique, éd Echeverria, tr. Parmentier, Vrin, Paris, 1995, $368 \mathrm{p}$.

\section{De l'esprit géométrique}

BLANCHÉ Robert, L'axiomatique, P.U.F., Paris, 1980, 110 p.

BLANCHÉ Robert, Le raisonnement, P.U.F., Paris, 1973, 264 p.

BLANCHÉ Robert, La logique et son histoire d'Aristote à Russell, Colin, Paris, 1970, 366 p.

BOREL Émile, «La définition en mathématiques », Le Lionnais, Les grands courants de la pensée mathématique, Blanchard, Paris, 1962, p. 24-34. 
CLERTÉ B. et LHOSTE-NAVARRE M., L'Esprit de la géométrie et De l'Art de persuader. Textes et commentaires, Éditions Pédagogique Moderne, Paris, 1979, 234 p.

DAVAL R. et GUILBAUD G.-T., Le raisonnement mathématique, P.U.F., Paris, 1945, 152 p.

GARDIS Jean-Louis, Le raisonnement par l'absurde, P.U.F., Paris, 1991, 206 p.

GRANGER Gilles-Gaston, «L'usage philosophique des mathématiques au XVII ${ }^{e}$ siècle », in Rashed Roshdi (dir.), Mathématiques et philosophie p. 287-301.

SCHOBINGER Jean-Pierre, Kommentar zu Pascals Reflexionen über die Geometrie im Allegemeinen, Schwabe, Basel, 1990.

TIMMERMANS Benoît, La résolution des problèmes de Descartes à Kant, P.U.F., Paris, 1995, 320 p.

\section{Coniques}

BELLIVIER Paul, « Le théorème de Pascal pour les coniques », Pascal et la géométrie, Cahiers pédagogiques de philosophie et d'histoire des mathématiques, fascicule 5, CRDP Rouen, 1993, p. 1-16.

BKOUCHE Rudolf, «La naissance du projectif : de la perspective à la géométrie projective », Rashed Roshdi (dir.), Mathématiques et philosophie, p. 239-285.

COLERUS Egmont, Du point à la quatrième dimension ou la géométrie pour tous, Flammarion, Paris, 1957, $348 \mathrm{p}$.

COMAR Philippe, La perspective en jeu. Les dessous de l'image, Découvertes, Gallimard, Paris, 1992, $128 \mathrm{p}$.

DHOMBRES J., « La culture mathématique au temps de la formation de Desargues : le monde des coniques », Dhombres et Sakarovitch, Desargues en son temps, p. 55-86.

DORRIE Heinrich, 100 great problems of elementary mathematics. Their history and solution, Dover, New York, 1965, 394 p.

HARA Kokiti, « Nouvelles observations sur les écrits mathématiques de Pascal », Historia Scientiarum, 26, 1984, p. 1-17.

KLINE Morris, Mathematics for the Nonmathematicain, Dover, New York, 1967, 642 p.

KNOBLOCH Eberhard, «L'analogie et la pensée mathématique », Rashed Roshdi (dir.), Mathématiques et philosophie, p. 217-237.

MESERVE Bruce M., Fundamental concepts of geometry, Dover, New York, 1983, 322 p.

MESNARD Jean, « Desargues et Pascal », Dhombres et Sakarovitch, Desargues en son temps, p. 87-100.

MESNARD Jean, " Point de vue et perspective dans les Pensées de Pascal », Courrier du Centre International Blaise Pascal, $\mathrm{n}^{\circ}$ 16, 1994, p. 3-8.

SERRES Michel, Le système de Leibniz et ses modèles mathématiques, t. 2, P.U.F., Paris, 1968, p. 668-671.

TATON René, « Desargues et le monde scientifique de son époque », Dhombres et Sakarovitch, Desargues en son temps, p. 23-54.

TATON René et FLOCON Albert, La perspective, P.U.F., Paris, 1970, 128 p.

WELLS David, Le Dictionnaire Penguin des curiosités géométriques, Eyrolles, Paris, 1996, 272 p. 


\section{Triangle arithmétique}

CARAMATIE Marie-Catherine, «Traité du triangle arithmétique », Pascal et la géométrie, Cahiers pédagogiques de philosophie et d'histoire des mathématiques, fascicule 5, CRDP Rouen, 1993, p. 17-42.

COSTABEL Pierre, « Leibniz et les séries numériques », in Heinekamp Albert et Mettler Dieter (dir.), Leibniz à Paris (1672-1676), I, les Sciences, p. 81-102.

DESCOTES Dominique, « La responsabilité collective dans les Provinciales », in Duchêne Roger, L'Imposture littéraire dans les Provinciales de Pascal, Publ. Univ. de Provence, Aix, 1985, (2 éd.), p. 350-373.

EDWARDS A. W. F., Pascal's Arithmetical Triangle, Oxford University \& Charles Griffin, New York \& Londres, $1987,174 \mathrm{p}$.

Cet ouvrage contient une bibliographie sur le Triangle arithmétique, les ordres numériques et la "géométrie du hasard».

GROUPE INTER-IREM ÉPISTEMOLOGIE ET HISTOIRE DES MATHÉMATIQUES, La Rigueur et le calcul. Documents historiques et épistémologiques, CEDIC, Paris, 1982, 280 p.

LAM Lay Yong, « The chinese connection between the Pascal Triangle and the solution of numerical equations of any degree », Historia mathematica, 7, 1980, p. 407-427.

ORE Oystein, Number theory ans its history, Dover, New York, 1976, 370 p.

ORE Oystein, Initiation à la théorie des nombres, Dunod, Paris, 1970, 154 p.

SERRES Michel, « Leibniz retraduit en langue mathématique », Hermès III, La Traduction, p. 127 sq.

SERRES Michel, « De l'Art combinatoire », Leibniz. Aspects de l'homme et de l'œuvre, AubierMontaigne, Paris, 1968, p. 119-130.

SINGMASTER David, «Quelques divertissements numériques », La Recherche, 278, juillet-août 1995, p. 818-823. Voir p. 823, problème non résolu nº 10.

WELLS David, Le dictionnaire Penguin des nombres curieux, Eyrolles, Paris, 1995.

\section{Ordres numériques}

DAHAN-DALMEDICO Amy et PEIFFER Jeanne, Routes et dédales, Paris-Montréal, 1982, 284 p.

DHOMBRES J., DAHAN-DALMEDICO A., BKOUCHE R., HOUZEL C., GUILLEMOT M., Mathématiques au fil des âges, Gauthier-Villars, Paris, 1987, 328 p.

FOURREY Émile, Récréations mathématiques, Vuibert, Paris, 1899, éd. 1994 augmentée d'une étude de Jean-Louis Nicolas, 264 p.

ITARD Jean, arithmétique et théorie des nombres, P.U.F., Paris, 1973, 128 p.

MESANRD Jean, « Nombres et textes figurés chez Pascal », XVII ${ }^{e}$ Siècle, 177, oct.-déc. 1992, 4, p. 521-532. 


\section{De numeris multiplicibus}

CHABERT Jean-Luc, BARBIN Évelyne, GUILLEMOT Michel, MICHEL-PAJUS Anne, BOROWCZYK Jacques, DJEBBAR Ahmed, MARTZLOFF Jean-Claude, Histoire d'Algorithmes. Du caillou à la puce, Belin, Paris, 1994, 592 p.

HARA Kokiti, « Nouvelles Observations sur les Écrits mathématiques de Pascal (II) », Historia Scientiarum, 27, 1984, p. 11-25.

\section{Combinaisons}

COUMET Ernest, « Mersenne : dénombrements, répertoires, numérotations de permutations », Mathématiques et sciences humaines, 38, 1972, p. 5-37.

COUMET Ernest, « Mersenne : dictions nouvelles à l'infini », XVII ${ }^{e}$ Siècle, 109, 1975, p. 1-32.

COUMET Ernest, « Cryptographies est numérotations », Annales, 5, 1975, p. 1007-1027.

MEURILLON Christian, «Les combinaisons pascaliennes ou les avatars de la pensée ternaire », Équinoxe, 6, Rinsen Books, Kyoto, 1990, p. 49-68.

\section{Sommation des puissances numériques}

BOLL Marcel, Histoire des mathématiques, P.U.F., Paris, 1979, 13éd., 128 p.

KLINE Morris, Mathematical thought from ancient to modern times, Oxford Univ. Press, 1972, 3 vol. MAHONEY Michael Sean, The mathematical Career of Pierre de Fermat, 1601-1665, $2^{\mathrm{e}}$ éd., Princeton Univ. Press, 1994, 432 p.

\section{Partis}

ABOUT Pierre-José et BOY Michel, La Correspondance de Blaise Pascal et de Pierre de Fermat. La Géométrie du Hasard ou le début du calcul des probabilités, Cahiers de Fontenay, 32, Fontenayaux-Roses, Paris, 1983, 92 p.

BOUCHAUD Jean-Philippe, « Les lois des grands nombres », La Recherche, 278, juillet-août 1995, p. $784-788$.

BOURSIN Jean-Louis et CAUSSAT Pierre, Autopsie du Hasard, Bordas, Paris, 1970, 334 p.

BOURSIN Jean-Louis, Les Structures du Hasard, Seuil, Paris, 1966, 192 p.

CHEVALLEY Catherine, Pascal. Contingence et probabilités, P.U.F., Paris, 1995, 128 p.

COUMET Ernest, « La Théorie du hasard est-elle née par hasard ? », Annales, mai-juin 1970, p. 574-598.

COUMET Ernest, « Sur « le calcul ès jeux de hasard » de Huygens : dialogue avec les mathématiques français (1655-1657) », Huygens et la France, Vrin, Paris, 1982, 268 p., p. 123-138. GROUPE INTER-IREM ÉPISTEMOLOGIE ET HISTOIRE DES MATHÉMATIQUES, La Rigueur et le calcul. Documents historiques et épistémologiques, CEDIC, Paris, 1982, 280 p.

GUILBAUD G. Th., « La Règle des partis et la ruine des joueurs », M. S. H., 9, 1969.

JACQUARD Albert, Les Probabilités, P.U.F., Paris, 1974, 128 p. 
KASNER E. et NEWMAN J., Les Mathématiques et l'Imagination, Payot, Paris, 1970, 252 p.

LAPLACE Pierre-Simon, Essai philosophique sur les Probabilités, Bourgois, Paris, 1986, 314 p.

LEIBNIZ G. W., L’Estime des apparences, éd. Parmentier, Vrin, Paris, 1995, 474 p.

Pascal et les Probabilités, Cahiers pédagogiques de Philosophie et d'Histoire des Mathématiques, CRDP Rouen, 1993, 120 p.

RAYMOND Pierre, De la combinatoire aux probabilités, Maspero, Paris, 1975, 174 p.

RUHLA Ch., La Physique du hasard de Blaise Pascal à Niels Bohr, Hachette-CNRS, Paris, 1989, $270 \mathrm{p}$.

SERIS Jean-Pierre, La théorie des jeux, P.U.F., Paris, 1974.

SHEYNIN O. B., " On the Prehistory of the Theory of Probability », Archive for History of exact Science, Berlin-Heidelberg-New York, vol. 12, 1974, p. 97-141.

THIROUIN Laurent, Le hasard et les règles. Le modèle du jeu dans la pensée de Pascal, Vrin, Paris, 1991, 222 p.

NB : Le dossier de Pour la Science sur Le Hasard, hors-série d'avril 1996, ne cite pas le nom de Blaise Pascal.

\section{Carrés magiques}

ANDREWS W. S., Magic Squares and Cubes, Dover, New York, 1960, 420 p.

DARRIULAT Jacques, L'Arithmétique de la grâce. Pascal et les carrés magiques, L’Âne d'Or, Les BellesLettres, Paris, 1994.

KRAITCHIK M., Traité des carrés magiques, Gauthier-Villars, Paris, 1930, 108 p.

\section{Indivisibles et infini}

BLAY Michel, Les raisons de l'infini. Du monde clos à l'univers mathématique, Gallimard, Paris, 1993, 258 p. Comprendre l'infini, Sciences et Avenir, Hors-série, nº 105, mars 1996, 100 p.

BODART Roger, «Les espaces infinis (de Saint Augustin à Pascal) », Chronique de Port-Royal, 20-21, 1972, p. 131-135.

BOLL Marcel, Histoire des mathématiques, P.U.F., Paris, 1979 (13éd.), 128 p.

BOLZANO Bernard, Les paradoxes de l'infini, éd. H. Sinaceur, Seuil, Paris, 1993, 194 p.

BURBAGE Frank et CHOUCHAN Nathalie, Leibniz et l'infini, P.U.F., Paris, 1993, 126 p.

CIFOLETTI Giovanna Cleonice, La méthode de Fermat : son statut et sa diffusion, Cahiers d'Histoire et de Philosophie des sciences, n 33, 1990, Belin, Paris, 244 p.

COHN Jonas, Histoire de l'infini, Le Problème de l'infini dans la pensée occidentale jusqu'à Kant, Cerf, Paris, 1994, 266 p.

COLERUS Egmont, De la table de multiplication à l'intégrale, Flammarion, Paris, 1952, 320 p.

DELACHET André, L'analyse mathématique, P.U.F., Paris, 1977 (7éd.), 128 p.

DELACHET André, Calcul différentiel et intégral, P.U.F., Paris, 1980, 128 p. 
DESCOTES Dominique, « Espaces infinis égaux ou finis », in Montandon Alain (dir.), Le grand et le petit, CRDP, Clermont-Ferrand, 1990, p. 41-68.

DESCOTES Dominique, «Sur quelques signes géométriques de Dieu chez le P. Mersenne et Pascal », in Demerson Geneviève et Dompnier Bernard (dir.), Les Signes de Dieu, Faculté des Lettres, Clermont-Ferrand, 1993, p. 269-284.

DESCOTES Dominique, "Disproportion de l'homme : de la science au poème ", in Goyet Thérèse (dir.), L’Accès aux Pensées de Pascal, Klincksieck, Paris, 1993, p. 145-162.

DHOMBRES J., DAHAN-DALMEDICO A., BKOUCHE R., HOUZEL C., GUILLEMOT M., Mathématique au fil des âges, Gauthiers-Villars, Paris, 1987, 328 p.

GANDT François (de), « Mathématiques et réalité physique au XVII ${ }^{\mathrm{e}}$ siècle (de la vitesse de Galilée aux fluxions de Newton)», Penser les mathématiques, Seuil, Paris, 1982, p. 167-194.

GARDINER A., Infinite processes. Background to analysis, Springer-Verlag, New York, Heidelberg, Berlin, 1982, 306 p.

HILDEBRAND Stefan et TROMBA Anthony, Mathématiques et formes optimales, Pour la Science, Belin, Paris, 1986, 180 p.

HOUZEL Christian, OVAERT Jean-Louis, RAYMOND Pierre, SANSUC Jean-Jacques, Philosophie et calcul de l'infini, Maspero, Paris, 1976, 240 p.

LE LIONNAIS, Les grands courants de la pensée mathématique, Blanchard, Paris, 1962, 560 p. Le nombre $\pi$, Association pour le Développement de la Culture Scientifique, Amiens, 1992, 314 p. MAGNARD Pierre, «L'infini pascalien », Revue de l'Enseignement philosophique, 31 e année, 1, p. 2-16.

MESNARD Jean, Les Pensées de Pascal, 2e éd., SEDES-CDU, Paris, 1993, p. 71.

MONNOYEUR Françoise (dir.), Infini des mathématiciens, infini des philosophes, Belin, Paris, 1992, $216 \mathrm{p}$.

REDONDI Pietro, Galilée hérétique, NRF, Gallimard, Paris, 1983, 488 p.

\section{Lettres de A. Dettonville}

BARON Margaret E., The Origins of the infinitesimal calculus, Dover, New York, 1969, 304 p.

BELAVAL Yvon, Leibniz. Initiation à sa Philosophie, Vrin, Paris, 1975, p. 86-88.

BELAVAL Yvon, Leibniz critique de Descartes, NRF, Gallimard, Paris, 1960, 560 p.

BOS H. J. M., «L'élaboration du calcul infinitésimal, Huygens entre Pascal et Leibniz », Huygens et la France, Vrin, Paris, 1982, 268 p., p. 115-122.

BOURBAKI Nicolas, Éléments d'histoire des mathématiques, Masson, Paris, 1984, 376 p.

BOUVERESSE Renée, Leibniz, P.U.F., Paris, 1994, 128 p.

CLERO Jean-Pierre et le REST Évelyne, La Naissance du calcul infinitésimal au XVII ${ }^{e}$ siècle, Cahiers d'Histoire et de Philosophie des Sciences, 16, 1980, 194 p.

DE GANDT François, «Ébauche d'une notice sur momentum », Document pour l'histoire du vocabulaire scientifique, $\mathrm{n}^{\circ}$ 1, Publications de l'Institut de la Langue Française, CNRS, 1980, p. 1-20. 
DESCOTES Dominique, « Documents relatifs aux Lettres de A. Dettonville, I, Pascal et le Père Tacquet », Courrier du Centre International Blaise Pascal, 14, 1992, p. 18-53.

DESCOTES Dominique, « Aux origines du Triangle caractéristique », L’Âne, 54-55, été 1993, p. $43-45$.

GRANGER Gilles-Gaston, Langages et épistémologie, Klincksieck, Paris, 1979, 226 p.

« La cycloïde : méthode de recherche du centre de gravité », Pascal et la géométrie, Cahiers

pédagogiques de philosophie et d'histoire des mathématiques, fascicule 5, CRDP Rouen, 1993, p. 27-42.

LEIBNIZ G. W., Naissance du calcul différentiel, éd. Parmentier, Vrin, Paris, 1989, 500 p.

Pascal et la géométrie, Cahiers pédagogiques de philosophie et d'histoire des mathématiques, 5, CRDP

Rouen, 1993, 128 p.

\section{La roulette}

1588-1988. Quatrième centenaire de la naissance de Marin Mersenne, Univ. du Maine, 1994, 206 p.

APERY R., CAVEING M., DIEUDONNÉ J., MANDELBROT B., THOM R., et alii, Penser les mathématiques, Seuil, Paris, 1982, $280 \mathrm{p}$.

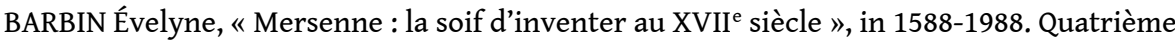
centenaire de la naissance de Marin Mersenne, p. 27-46.

De points en courbes. Histoire, construction, utilisation pédagogique des courbes mathématiques célèbres, CRDP Dijon, 1987, 264 p.

TAILLE Jean, Courbes et surfaces, P.U.F., Paris, 1953, 128 p. (voir p. 96 sur la cycloïde).

\section{Machine arithmétique}

Baisse Pascal « Auvergnat ». La famille à l'œuvre, Musée d'Art de Clermont-Ferrand et Amis CIBP, Clermont-Ferrand, 1981, 112 p.

BRABANDERE Luc de, Calculus. Les machines du calcul non électriques, Mardaga, Liège, sd 1994.

De la machine à calculer de Pascal à l'ordinateur : 350 ans d'informatique, Exposition Musée nationale des Techniques, CNAM, Paris, 26 avril-23 septembre 1990, Paris, 1990, 64 p.

DESCOTES Dominique, « Pascal et le marketing », Mélanges offerts au Professeur Maurice Descotes, Univ. de Pau et des pays de l'Adour, Pau, 1988, p. 141-162.

ELLENBERGER Michel et COLLIN Marie-Marthe, La Machine à calculer de Blaise Pascal, Nathan, Paris, 1993, $80 \mathrm{p}$.

GOYET Thérèse, «Le manuscrit Usage de la Machine », Courrier du Centre International Blaise Pascal, $\mathrm{n}^{\circ} 4,1982$, p. 6-11.

GOYER Thérèse, "Quelques séquences de l'exposition », Courrier du Centre International Blaise Pascal, 3, 1981, p. 3-13.

GRAF Klaus-D., « Calculating machines in China and Europe in the 17th century. - The western view », 13th World Computer Congress, 94, vol. 2, K. Brunnstein ans E. Raubold éd., Elsevier Science B. V. (North-Holland), 1994. 
GRAF K.-D., BAI Shangshu, LI Di, « The calculating machines stored in the Palace Museum in Beijing, their structure and algorithm », 13th World Computer Congress 94, vol. 2, K. Brunnstein ans E. Raubold ed., Elsevier Science B. V. (North-Holland), 1994.

IFRAH Georges, Histoire universelle des chiffres, Robert Laffont, 2 vol. , Paris, 1994, p. 496 sq.

Katalog der Sammlung historischern rechenmaschinen und Recenhislfsmittel, Bonn, Forshunginstitut für Diskrete mathematik, sd.

KORTE Bernhardt et KERHIBAUD Annegret, Pioniere des industriellen Revolution und Rechenmaschinen des 20. Jahrhunderts, DMV, 2/1993, p. 8-20.

KORTE Bernhardt, Zur geschichte des maschinellen rechnens, Bonn, Bouvier, 1981.

MARGUIN Jean, Histoire des instruments et machines à calculer. Trois siècles de mécanique pensante, 1642-1942, Hermann, Paris, 1994.

MEURILLON Christian, «La Machine arithmétique à la genèse des ordres pascaliens ", La Machine dans l'imaginaire (1650-1800), Revue des Sciences Humaines, LVIII, 186-187, avril-octobre 1982, p. $147-158$.

MOURLEVAT Guy, «L'artifice du mouvement de la machine arithmétique », Courrier du Centre International Blaise Pascal, 11, 1989, p. 28-31.

\section{Pascal physicien}

\section{Études générales}

BEAULIEU Armand, Mersenne. Le grand Minime, Fondation Nicolas-Claude Fabri de Peiresc, Bruxelles, diff. Les Belles-Lettres, 1996, 380 p.

BERROIR André, La météorologie, P.U.F., Paris, 1991, 128 p.

Blaise Pascal « Auvergnat ». La famille à l'œuvre, Musée d'Art de Clermont-Ferrand, 6 octobre-8 novembre 1981, Amis CIPB, Clermont-Ferrand, 1981, 112 p.

BLAMONT Jacques, Le chiffre et le songe. Histoire politique de la découverte, Odile Jacob, Paris, 942 p.

DE GANDT François, L'œuvre de Torricelli : science galiléenne et nouvelle géométrie, Publications de la Faculté des Lettres et Sciences humaines de Nice, $n^{\circ} 32$, (1 ${ }^{\text {re }}$ série), Nice, 1987, 248 p.

CHEVALLEY Catherine, « Nature et loi dans la philosophie moderne », in Kambouchner Denis, Notions de Philosophie, I, Folio, Galimard, Paris, 1995, p. 127-230.

DESCOTES Dominique, « Aspects littéraires des œuvres de Pascal sur le vide et d'hydrostatique », Équinoxe 6, été 1990, Rinsen Books, p. 31-48.

FANTON D'ANDON Jean-Pierre, L'Horreur du Vide, CNRS, Marseille, Paris, 1978, 156 p.

GRENET Micheline, La passion des astres au XVII siècle. De l'astrologie à l'astronomie, Hachette, Paris, 1994, 296 p.

GUENANCIA Pierre, Du vide à Dieu. Essais sur la physique de Pascal, Maspero, Paris, 1976, 342 p.

HOGBEN Launcelot, La science pour tous, Payot, Paris, 1946, 2 vol.

KOYANAGI Kimiyo, (« Les deux lettres de Torricelli et les recherches de Pascal »), Kagakusi Kenkiu, Journal of History of Science, Japon, Series 2, vol. 28, n 170, 1989, p. 65-79, et n 171, 1989, p. 129-134 (en japonais). 
KOYANAGI Kimiyo, (De l'intuition à l'affirmation : cheminement de Pascal jusqu'à l'achèvement de ses Euvres de physique), Japon, 1992, 26-470 p. (en japonais).

KOYANAGI Kimiyo, « De l'intuition à l'affirmation : cheminement de Pascal jusqu'à l'achèvement de ses Euvres de physique ", Études de Langue et Littérature françaises, $n^{\circ}$ 60, Société Japonaise de Langue et Littérature françaises, Tokyo, 1992, p. 44-56.

KOYANAGI Kimiyo, ( Formation du Traité : Expériences nouvelles touchant le vide. Petit, Pierius, Guiffart, Roberval, Descartes, Mersenne, Pascal »), Études de langue et littérature françaises, $\mathrm{n}^{\circ} 67$, 1995, p. 3-15 (en japonais).

KOYANAGI Kimiyo, («Les expériences de Pascal faites à Rouen. De la signification des expériences de Berti, Torricelli et Pascal. Dernière partie »), The Journal of the Faculty of Foreign Studies, Aichi Prefectural University, $n^{\circ} 25,1993$, p. 205-239, (en japonais).

KOYANAGI Kimiyo, («L'art de la controverse dans l'œuvre physique de Pascal »), Bulletin annuel de la Société de Langue et Littérature Françaises du Chûbu, 1993, p. 9-23 (en japonais).

LARRAS Jean, L'hydraulique, P.U.F., Paris, 1974, 128 p.

LICOPPE Christian, La formation de la pratique scientifique. Le discours de l'expérience en France et en Angleterre (1630-1820), Éditions La Découverte, Paris, 1996, 346 p.

MESNARD Jean, « Pascal et Copernic », Avant, avec et après Copernic, Blanchard, Paris, p. 241-249.

NORDON Marcel, Histoire de l'hydraulique, I, L'Eau conquise, 1991 ; II. L'Eau démontrée, 1992, Masson, Milan-Paris-Barcelone-Bonn, 2 vol.

SANDORI Paul, Petite logique des forces. Constructions et machines, Seuil, Paris, 1983, 204 p.

SHAPIN Steven et SCHAFFER Simon, Léviathan et la pompe à air. Hobbes et Boyle entre science et politique, Éditions la Découverte, Paris, 1993, 462 p.

\section{Expérience du vide dans le vide}

KOYANAGI Kimiyo, « Pascal et l'expérience du vide dans le vide ", Japanese Studies in the History of Science, 17, 1978, p. 105-127.

KOYANAGI Kimiyo et DESCOTES Dominique, « La reconstitution de l'expérience du vide dans le vide ", Courrier du Centre International Blaise Pascal, 11, 1989, p. 2-24.

\section{Grande expérience de l'équilibre des liqueurs}

BEN-DOV Yoav, Invitation à la physique, Points, Seuil, 1995, $210 \mathrm{p}$.

Blaise Pascal. Comment a-t-il démontré l'existence de la pression atmosphérique ?, Les Cahiers de Science et Vie, série Les grandes expériences de la Physique, Hors-série, nº 27, juin 1995.

"L'expérience du Puy-de-Dôme par l'ordinateur », Courrier du Centre International Blaise Pascal, $\mathrm{n}^{\circ} 10,1988$, p. 27.

MATTON Sylvain, « La grande expérience » du Puy-de-Dôme revisitée : à propos de la Science naturelle de G. B. de Saint Romain et de ses théories alchimiques », Chrysopoeia, II, fasc. 4, octobre-décembre 1988, p. 305-364. 


\section{Traité de la pesanteur de la masse de l'air}

LEVALLOIS Jean-Jacques, « Picard géodésien », in PICOLET Guy (dir.), Jean Picard et les débuts de l'astronomie de précision au XVII siècle, éd. CNRS, Paris, 1987, 382 p. (voir p. 227-246).

INDEX

Mots-clés : Pascal, bibliographie

Keywords : Pascal, bibliography

\section{AUTEUR}

DOMINIQUE DESCOTES

CERHAC, Université Blaise Pascal 